\author{
微流控技术在微/纳米材料合成中的研究进展 \\ 卢佳敏 ${ }^{a}$ 王慧峰 $a, c$ 潘建章 $a, b, c$ 方群*, $, a, b, c$ \\ ( ${ }^{a}$ 浙江大学 化学系微分析系统研究所 杭州 310058) \\ ( $b$ 浙江大学 浙江省激发态材料合成与应用重点实验室 杭州 310027) \\ (c 浙江大学 杭州国际科创中心 杭州 311200)
}

\begin{abstract}
摘要 微/纳米材料因其尺度的微小而具有异于宏观材料的特殊性质, 在多个领域都有着丰富的应用. 近年来, 微流控 技术因其微量、高效、高通量、微型化、集成化和自动化等独特的优势在微/纳米材料的合成中引起了广泛的关注. 本 综述从微反应器的结构形式及反应方式两个角度进行分类, 介绍了微流控技术在无机材料、有机材料和复合材料中的 具体应用, 并对该领域未来的主要发展趋势进行了展望. 微流控技术为微/纳米材料的合成提供了新的思路和方法, 在 工业生产和学术研究中都蕴含着丰富的可能性和巨大的潜力.
\end{abstract}

关键词＼cjkstart微流控技术; 流动化学; 微/纳米材料; 材料合成; 微反应器

\title{
Research Progress of Microfluidic Technique in Synthesis of Micro/Nano Materials
}

\author{
Jia-Min $\mathrm{Lu}^{a} \quad$ Hui-Feng Wang ${ }^{a, c} \quad$ Jian-Zhang Pan ${ }^{a, b, c} \quad$ Qun Fang*,a,b,c \\ ( ${ }^{a}$ Institute of Microanalytical Systems, Department of Chemistry, Zhejiang University, Hangzhou 310058, China) \\ ( ${ }^{b}$ Key Laboratory of Excited-State Materials of Zhejiang Province, Zhejiang University, Hangzhou 310027, China) \\ ( ${ }^{c}$ Hangzhou Innovation Center, Zhejiang University, Hangzhou 311200, China)
}

\begin{abstract}
Micro/nano materials have unique properties that are different from macro materials because of their small scale, and these materials have diverse applications in many fields. In recent years, with the unique advantages of low consumption, high efficiency, high throughput, miniaturization, integration and automation, microfluidic technique has aroused widespread concerns in the synthesis field of micro/nano materials. In this review, the commonly used microreactors are classified from two perspectives of structural form and reaction method, and the application of microfluidic technique in the synthesis of micro/nano materials including inorganic materials, organic materials and composite materials are introduced. In addition, the main development trends in the future are prospected in this review. Microfluidic technique has provided new ideas and methods for the synthesis of micro/nano materials, which has rich possibilities and great potentials in both industrial production and academic research.
\end{abstract}

Keywords microfluidic technique; flow chemistry; micro/nano material; material synthesis; microreactor

\section{1 引言}

微/纳米材料是指尺度在微米或纳米级的材料. 相 比于宏观尺度的材料, 微/纳米材料具有小尺寸效应、表 面效应、宏观量子隧道效应等特殊性质. 目前, 微/纳米 材料已被应用于传感 ${ }^{[1-3]}$ 、能量存储与转换 ${ }^{[4-6]}$ 、照明与 显示 ${ }^{[7-8]}$ 、生物医药 ${ }^{[9-12]}$ 与检测 $[13]$ 、成像 ${ }^{[14]}$ 和催化 ${ }^{[15-16]}$ 等多个领域.

对于具有特定功能的高质量微/纳米材料的合成而 言, 精确控制反应参数(如: 试剂的浓度、比例、反应的 温度和压力等)和步骤(如: 试剂的分离和混合等)是很重 要的 ${ }^{[17]}$. 目前, 传统且常用的批处理合成法可以生产出 质量在克级的微/纳米材料 ${ }^{[18]}$, 但仍存在混合不够均
匀、传质传热效率较低、对合成条件的控制不够精确、 优化反应条件的过程耗时、耗样量大、后处理方法费时 费力以及成本较高等问题 ${ }^{[19]}$. 随着对微/纳米材料产物 的粒度、粒度分布、形状以及批次间可重现性等性能的 要求日益提高, 亟需开发能满足上述要求的新合成方 法.

微流控技术因其独特的优势, 已经引起了生物传

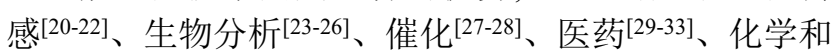
材料合成 ${ }^{[34]}$ 等各个领域学者的广泛关注. 微流控技术 在微/纳米材料合成领域的应用最早出现于 20 世纪 90 年代. 2002 年, Edel 等 ${ }^{[35]}$ 使用微流控技术代替批量合成 方法合成纳米晶体. 自此, 微流控技术的独特优势吸引 了众多科研人员的目光, 逐渐被广泛应用于高质量微/

\footnotetext{
* E-mail: fangqun@zju.edu.cn

Received March 9, 2021; published May 14, 2021.

Project supported by the National Natural Science Foundation of China (Nos. 21827806, 21974122).

项目受国家自然科学基金(Nos. 21827806, 21974122)资助.
} 
纳米材料的合成中.

在微/纳米材料的合成方面, 相比常规合成方法, 微流控技术的主要优势有: (1)微量: 微流控技术的耗样 量一般在 $10^{-9} \sim 10^{-12} \mathrm{~L}$ 范围内, 有效地降低了需要稀有 试剂的反应的成本 ${ }^{[36]}$. 对涉及不稳定或有害中间体的 反应而言, 在一定程度上降低了反应的危险性, 符合绿 色合成的发展趋势. (2)高效: 微流控技术所使用的微反 应器尺度微小, 因此微反应器中的传质、传热速率明显 比常规反应器更快. 这一优势能使反应器中的混合更加 均一, 促使反应更加高效和快速地完成, 还能显著提高 对反应的控制力和对产品理化性质的调节能力 ${ }^{[37]}$. (3)高 通量: 通过多通道并行等方法能有效提高反应通量. 在 此基础上, 多通道并行的微反应器与在线检测等技术的 集成能实现对反应参数的高通量篎选. 这对合成反应前 期的路线设计和优化有着重要意义 ${ }^{[38]}$. (4)集成化: 微流 控技术能实现分离、混合、加热、加压、反应及检测等 操作单元的高度集成 ${ }^{[39]}$. 例如, 在线检测技术可以轻松 地在毫秒级的时间内提取多项重要的反应参数 ${ }^{[40]}$. (5) 自动化: 微流控技术与计算机和自动化等技术结合能使 合成系统的自动化程度明显提高, 随着人工操作的减 少, 实验人员在高温高压等复杂条件下面临的潜在风险 也相应减少 ${ }^{[41-42]}$. (6)智能化: 在人工智能等技术的支持 下，合成系统有望从实验数据中获得更加丰富的信息， 进行及时反馈并提供下一步合成建议, 帮助实验人员开 拓思路、提高效率. (7)易于扩大化: “数量放大” 方法 是指平行设置多个反应通道, 能成倍地提高反应通量, 从而将实验室中的微设备直接扩展运用于工业生产中.

在化学合成领域, 还有一个重要的概念—— “流动 化学” . 实际上, 这种通过将物料百入连续流动反应器 中混合并反应的化学合成方法在石油化工方面的应用 已经较为成熟, 有近百年的历史. 然而, 直到近 20 年, 流动化学才开始在微/纳米材料合成领域受到关注. 以 往的文献很少会对流动化学和微流控技术二者的概念 进行明确的区分, 但实际上它们之间存在一些区别：(1) 反应器的尺度不同: 微流控技术中使用的是通道尺度在 微米范围(数十 $\mu \mathrm{m}$ 至 $1 \mathrm{~mm}$ ) 的微型反应器, 一般用于生 产 $<100 \mathrm{~g}$ 的产品. 而流动化学中除了上述微型反应器 之外, 还有通道尺寸较大的反应器: 中型反应器的通道 尺度在毫米范围(1 $10 \mathrm{~mm})$, 一般用于生产 $<100 \mathrm{~kg}$ 的 产品; 大型反应器的通道尺度在厘米范围 $(1 \mathrm{~cm}$ 及以上), 年产量可达吨级 ${ }^{[43]}$. 中、大型反应器由于通道尺度较大, 传质传热速率以及混合的均一性低于微型通道反应器; 但同时, 大尺度通道中发生结垢或堵塞的可能性较小, 也更容易获得较大的产量. (2)反应的形式不同: 流动化 学合成通常基于 “流动” 形式进行; 而微流控技术的核 心则是 “对微量流体的操控”, 因此反应形式并不仅仅 限于流动, 还包括通过非流动形式进行化学合成的技术 (如间歇反应器等).
总而言之，上述诸多优势使微流控技术能够被广泛

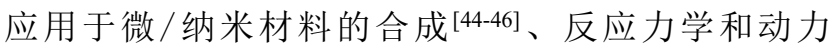
学 ${ }^{[47-49]}$ 、检测及高通量篮选 ${ }^{[50-52]}$ 等不同方面, 为高质量 微/纳米材料的相关研究提供了一条理想的新途径.

\section{2 基于微流控技术合成微/纳米材料的微反应 器}

广义的 “微反应器”一般包括微混合器、微反应器、 微加热单元、微控制器(传感器)等部分. 在文献中常用 的狭义的概念是指通道直径范围从数十 $\mu \mathrm{m}$ 至 $1 \mathrm{~mm}$ 的 反应器 ${ }^{[53]}$. 常见的基于微流控技术合成微/纳米材料的 微反应器按结构形式可分为微管路和微流控芯片反应 器; 按反应器中的反应方式可分为间歇微反应器和流动 (包括单相流动和多相流动)微反应器. 间歇微反应器又 可被称为批式反应器. 流动反应器中, 按微通道结构的 不同, 还可将微反应器分为开管式反应器(包括直管和 盘管)和填充管式反应器.

上述不同类型的微反应器均可以与光、电、热、压、 磁等各种功能模块中的一种或多种进行集成, 满足不同 实验对实验条件的需求.

此外, 在微反应器的微通道中进行高效混合是制备 可控微/纳米材料的基础. 不同于宏观体系, 微反应器中 通道尺度的显著降低使分子扩散效应的影响变得十分 显著, 利用此效应可获得较快的混合速度. 目前使用较 多的方法是对通道进行特殊设计以提高混合效率，例如 在通道内部设计人字形微结构等; 或者采用多相微流控 液滴体系, 利用微液滴内环流实现液滴组分的快速混 合. 微流控技术实现的毫秒或纳秒级混合有效提高了 微/纳米材料的可控性和均匀性, 并为材料合成带来了 新的可能性 ${ }^{[54]}$.

\section{1 微管路和微流控芯片反应器}

在实际应用中，具体合成所需的反应物和反应条件 不同, 对微反应器材料的要求也不同, 这些要求涉及反 应器的耐高温高压性质、溶剂相容性、光学透明性、对 试剂的渗透性、与泵和阀等元件的易集成性，以及适当 的表面化学性质等. 除了选择合适的微反应器的材质之 外, 科研人员往往还需要根据实际反应的特点选择合适 的反应器结构形式. 按微反应器的结构形式可以将用于 微/纳米材料化学合成的微反应器分为微管路和微流控 芯片反应器两大类 ${ }^{[55]}$ (图 1).

微管路反应器通常选择采用熔融石英、不锈钢、聚 四氟乙烯(PTFE)、四氟乙烯-全氟烷氧基乙烯基醚共聚 物(PFA)、聚醚醚酮(PEEK)等材质的毛细管，配合三通、 原、阀、加热模块等完成系统的构建 ${ }^{[56-57]}$. 微流控芯片 反应器一般根据反应需求选择合适的材质, 如玻璃、硅、 不锈钢、镍等无机材料, 或聚甲基丙烯酸甲酯(PMMA)、 聚二甲基硅烷(PDMS)和聚碳酸酯(PC)等聚合物材料, 
然后通过不同的微加工方法进行加工, 将涉及试剂添 加、混合、加热和冷却等多种化学过程的单元集成在一 个芯片上 ${ }^{[58]}$.
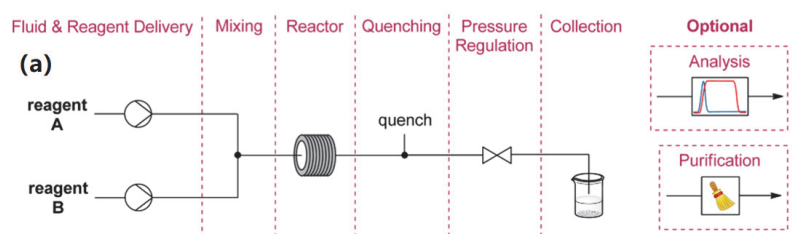

(b)

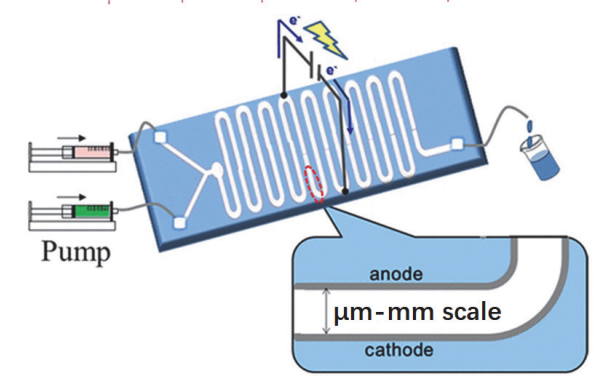

图 1 典型的微管路反应器( $(a)$ 和微流控芯片反应器(b)示意图 ${ }^{[56,58]}$

Figure 1 Schematic diagram of a typical micro-conduit based (a) microreactor and a microchip-based (b) microreactor ${ }^{[56,58]}$

微管路和微流控芯片反应器具有不同的优势和局 限性 ${ }^{[59]}$ : (1)微管路反应器的优势包括结构较简单、容易 搭建、各功能模块可按需灵活组装等; 然而其通道接口 的死体积较大, 所能实现的操作与微流控芯片反应器相 比也较为简单 ${ }^{[60]}$. (2)微流控芯片反应器的优势包括能在 更小的空间中集成较多元件、能实现较复杂的操作以及 能够通过设计不同构型的通道实现较快的传质传热等; 然而微流控芯片反应器的加工过程较为复杂, 成本较 高, 各功能模块的组装灵活性低于微管路反应器. 因此, 综合来看, 微管路和微流控芯片反应器也可以相互结合 使用, 扬长避短. 例如, 不同的功能模块可以被加工在 不同的芯片上, 各芯片之间通过微管路进行连接. 在实 际合成中, 研究人员可以按照具体合成步骤的需求对不 同芯片模块进行组合, 这样的系统具有较高的灵活性和 通用性 ${ }^{[61]}$.

此外, 在芯片反应器中, 基于数字微流控技术的微 反应器是比较特别的一类. 在该类系统中, 通过施加适 当的电压即可控制液滴在开放的电极阵列上的移动, 从 而完成预浓缩、反应、净化和产品回收等步骤[62-65].

\section{2 间歇微反应器和流动微反应器}

在实际操作中, 科研人员除了应该关注微反应器的 形式, 还需选择反应的具体方式. 用于微/纳米材料合 成的微反应器可以按反应方式分为间歇和流动微反应 器, 其中流动微反应器又包括单相流动和多相流动微反 应器两类.

在间歇反应器中, 反应在微体积的固定容器中 (如 96、384、1536 孔板或阵列排列的微量瓶等)通过非流动 的方式进行(图 2(a) $)^{[66-67]}$. McNally 等[68]利用模块化全自 动合成 Chemspeed 系统, 发现了一种新的氧化还原催化
的 $\mathrm{C}-\mathrm{H}$ 芳基化反应. Chemspeed 系统可以完成包括试 剂制备(液体和固体)、多步合成、后处理、纯化、分析 和产品转移存储等多个步骤, 其特点是对固体试剂的操 作能够像对液体试剂的操作一样方便而准确. 与流动微 反应器相比, 间歇微反应器易于实现并行的高通量篮 选. 由于间歇微反应器中的合成不是在微通道中通过流 动实现，因此不存在通道堵塞或结垢等风险，但存在传 质传热速率较慢以及多次加样与取样比较困难等问题.

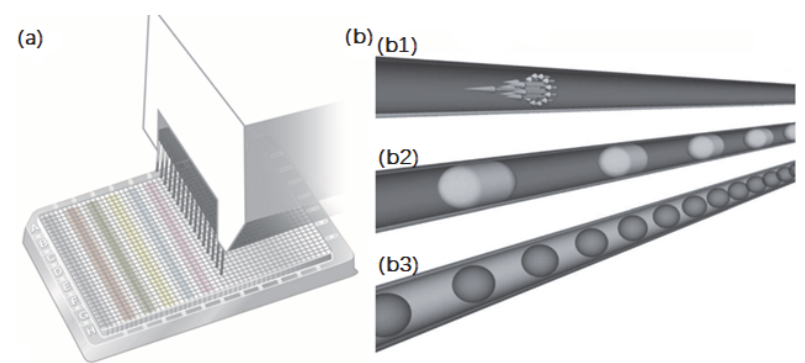

图 2 典型的间歇微反应器 ${ }^{[66]}$ (a) 和流动微反应器 ${ }^{[59]}$ (b) 结构示意图. (b1)连续流动模式; (b2)分段流动模式; (b3)液滴流动模式

Figure 2 Schematic diagram of a typical batch microreactor ${ }^{[66]}$ (a) and a continuous-flow microreactor ${ }^{[59]}$ (b). (b1) Continuous flow mode; (b2) segmented flow mode; (b3) droplet flow mode

单相流动微反应器仅涉及单一互溶相的连续流 动 ${ }^{69]}$ (图 2(b1)). 近年来, 单相流动微反应器的应用十分 广泛，但仍面临一些问题: (1)液流中的物质在管壁上的 沉积会影响后续产物的质量. 此问题虽可通过优化微通 道设计和调节流量等方法得到一定程度的改善，但仍有 待进一步研究 ${ }^{[70]}$. (2)在连续流中, 不同位置的流体速度 不同，这样的 “速度分散”使流体在反应区域的停留时 间不同，导致产物可能出现粒径分散和性能下降等问 题 ${ }^{[71-72]}$.

多相反应则涉及两个及以上的不混溶相, 包括液液、气-液、液-固、气-液-固等多种形式(图 2(b2), (b3)). 目前，应用最广泛的多相流动系统采用的液-液或气-液 分段流动模式, 通过引入与试剂相不混溶的载液相将试 剂相分隔成互不接触的液段. 其主要优势有: (1)每个液 段以基本相同的流速通过反应区域，减少速度分散；(2) 不同液段相当于互相独立的微反应器, 反应物质理论上 不与管壁接触，减少了结垢与交叉污染的风险; (3)短时 间内可按需生成不同条件的液段，结合在线检测技术有 望实现反应条件的高通量笁选 ${ }^{[73-74]}$. 分段流动模式仍面 临很多挑战，例如，需维持稳定的流体分布以及高温反 应需用高沸点液相以保证不发生混溶等.

固-液多相流动微反应器有着在后处理中易于分离 的特点, 因此对工业过程中涉及非均相催化剂的反应具 有特别的吸引力. 例如, Coley 等 ${ }^{[75-76]}$ 以 $\mathrm{Ru}(\mathrm{bpy})_{3} \mathrm{Cl}_{2}$ 作 为光催化剂研究了苯基硼酸转化为相应酚的反应以及 光氧化还原 $\mathrm{Ir}-\mathrm{Ni}$ 双催化体系. 催化剂被固定在反应通 道内特定区域的载体中, 有效摩尔浓度明显提高, 不但 加速了化学转化, 而且无需催化剂回收步骤. 然而, 催 化剂须定期重新活化或填充. 此外, 固相催化剂的更换 
操作较为麻烦.

对气-液多相流动系统而言, 微反应器可承受较高 的压力并降低高压气体的危害, 同时气体溶解度更好, 能改善界面混合并提高反应速率. 除了上文提到的分段 的气-液多相流之外, 还有一种比较特殊的套管式气-液 多相流动微反应器. Polyzos 等 ${ }^{[77]}$ 将 Teflon AF-2400 管置 于较大直径的 PTFE 外管中, 通过这种套管结构研究格 氏试剂的羧化反应. 具体来说, 液相在内管流动, 气体 则填充 PTFE 外管, 并通过分子扩散将反应性气体传输 到内管液流中. 此外, 通过套管式反应器还能得到吡

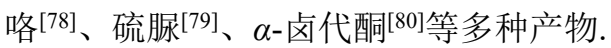

液滴多相流动微反应器中的试剂相以一系列离散 液滴的形式存在 ${ }^{[81]}$, 是分段流动模式中的一种特殊模 式 ${ }^{[82]}$. Shestopalov 等 ${ }^{[83]}$ 在 2004 年首次报道在液滴多相 流动微反应器中合成 $\mathrm{CdS}$ 量子点. 相比普通的分段流动 模式, 液滴多相流动模式最重要的优势是液滴与通道壁 理论上不发生接触, 可能发生沉积的物质被安全地包裹 在液滴内, 明显降低了通道壁被污染的风险 ${ }^{84-85]}$. 但液 滴系统对科研人员的操控技术和液滴生成环境等提出 了更高的要求. 此外, 液滴流动模式中还有一种比较特 别的振荡液滴流动微反应器, 通过在通道两端施加交替 的压力梯度实现微反应器通道中的一系列(或单个)液滴 的振荡. 反应所需的时间比连续流动和常规的液滴流动 模式所需的时间更长, 同时保留了较好的传质传热特 性, 与传统的连续多相流方法相比能进行更长时间的实 验研究.

\section{3 微流控技术在微/纳米材料化学合成中的应 用}

相比于宏观材料, 微/纳米尺度的材料显示出不同 的物理、化学、光学和热学性质. 基于微流控技术的微/ 纳米材料合成体系可以提供集成了设计、合成和检测等 多功能的一体化平台. 从物理和化学属性的角度, 材料 可以被分为无机材料、有机材料和复合材料三大类. 目 前, 微流控技术已被应用在这三类微/纳米材料的合成 中.

此外，微流控技术在化学合成方面也取得了显著的 进展. 例如, $\mathrm{Le}$ 等 ${ }^{[86]}$ 利用在微通道中的快速混合在不添 加稳定剂的情况下制备纳米药物. Chen 等 ${ }^{[87]}$ 采用了 Chemspeed SWING ISYNTH 系统以及定制的批式光反 应器, 在温和且实用的条件下实现了游离醇与芳基卤化 物的光催化脱羟甲基化芳基化, 通过铈和镍催化剂的协 同作用, 游离醇的 $\alpha-\mathrm{C}\left(\mathrm{sp}^{3}\right)-\mathrm{C}\left(\mathrm{sp}^{3}\right)$ 键可以选择性地裂 解并作为非常规合成子被应用于交叉偶联反应. Sun 等 ${ }^{88}$ 基于液滴微流体平台在皮摩尔水平上进行高通量 的三氟甲基化反应, 并通过在线电喷雾质谱高通量收集 数据, 以每秒 0.3 个样品的采集速率快速分析纳升大小 的反应液滴, 显著提高了反应及检测的效率. 这些进展
证明微流控技术在化学合成方面有着减少试剂量、提高 选择性、加速传质受限反应、提高安全性、反应器占地 面积小以及易于推广等独特的优势 ${ }^{[89]}$. 由于篇幅原因, 该部分内容在本文中不作进一步展开介绍.

本章主要从无机材料、有机材料和复合材料三类 微/纳米材料出发, 介绍微流控技术在微/纳米材料合成 领域的研究进展.

\section{1 无机微/纳米材料}

无机材料主要包括金属材料和无机非金属材料两 大类.

金属微/纳米材料被广泛应用在催化、能量存储、 医学和生物学等多个领域 ${ }^{[00]}$. 许多金属微/纳米材料的 性质会受到其形态的影响, 然而传统合成方法对产物形 态的控制仍不够理想. 例如, 一锅法中的形核和生长连 续发生、不易控制; 而在种子生长法中, 虽然形核和生 长是分开的, 但这种方法对反应条件的要求比较高, 重 现性和稳定性较差 ${ }^{[91]}$. 通过微流控技术, 科研人员能将 金属纳米颗粒的成核和生长过程分开，并很好地控制产 物的形态和尺度. Watt 等 ${ }^{[92]}$ 通过集成了在线紫外可见光 和近红外(UV-Vis-NIR)监测装置的微流控系统实时跟踪 金纳米棒的合成过程，并提出在合成种子后立即稀释能 有效改善老化的现象, 提高产物的质量. 基于微流控技 术, 目前研究人员已经成功合成出尺寸均匀、形状可控 的高质量金、银、铜和钯等多种金属微/纳米材料 ${ }^{[93-95]}$.

此外，微流控技术已经广泛应用于无机非金属材料 的合成、反应条件的优化以及反应动力学的机理研究等 方面 ${ }^{[96-98]}$. 量子点 (quantum dots, QDs)是一种尺寸小于 或相当于其激子波尔半径且形貌近似各向同性的半导 体纳米晶体, 具有光学性能可调、可溶、高光致发光、 高光谱纯度及高光化学稳定性等独特性能 ${ }^{[99]}$. Edel 等在 2002 年首次报道了基于连续微流控系统制备 CdS 量子 点, 此后微流控技术被越来越多地应用到量子点的合成 中. Lignos 等 ${ }^{[100]}$ 通过液滴多相流动微反应器合成了 $\mathrm{CsPbX}_{3}$ 量子点并在线检测其吸收和荧光光谱，只需数 毫升试剂和数小时的反应时间, 就能得到相当于 200 1000 个常规反应的信息(图 3(a)). Baek 等 ${ }^{[101]}$ 设计了最多 可串联六个不同功能芯片的多级微流控平台(芯片可根 据实际反应的需要进行灵活组装), 制备了高质量的 $\mathrm{InP} / \mathrm{ZnS} 、 \mathrm{InP} / \mathrm{ZnSe} 、 \mathrm{InP} / \mathrm{CdS}$ 和 $\mathrm{InAs} / \mathrm{InP}$ 等核/壳结构 量子点(图 3(b)).

此外，通过微流控技术还成功合成了包括碳酸钻

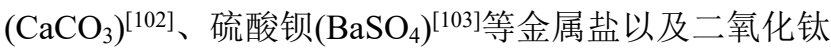
$\left(\mathrm{TiO}_{2}\right)^{[104]}$ 、氧化铁 $\left(\mathrm{Fe}_{3} \mathrm{O}_{4}\right)^{[105]}$ 、氧化锌 $(\mathrm{ZnO})^{[106]}$ 等金属 氧化物在内的多种无机非金属微/纳米材料.

\section{2 有机微/纳米材料}

一般而言，有机材料的合成具有一定的危险性. 2008 年, Yoshida 等 ${ }^{[107-108]}$ 将有机化学中已有的 “闪蒸化 
(a)

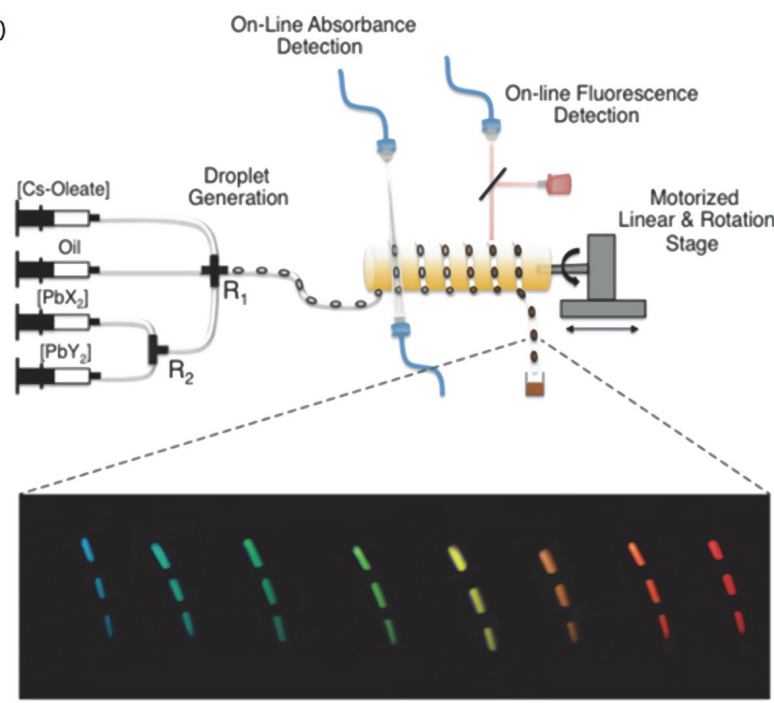

(b)

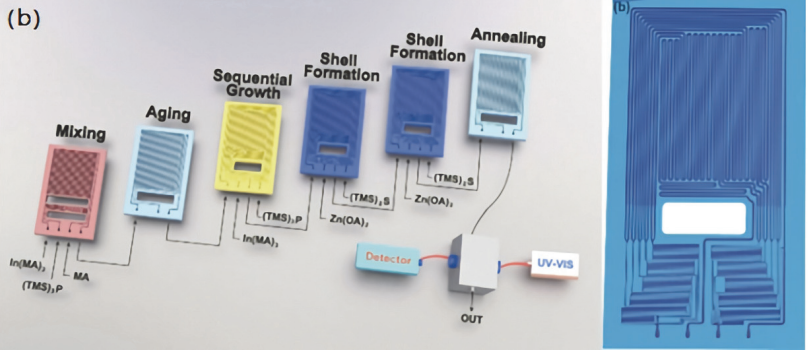

图 3 (a) 基于液滴微反应器的 $\mathrm{CsPbX}_{3}$ 量子点的合成 ${ }^{[100]}$; (b) 可串联 最多六个芯片反应器的多级微流控平台 ${ }^{[101]}$

Figure 3 (a) Synthesis of $\mathrm{CsPbX}_{3}$ quantum dots based on droplet microreactors $^{[100]}$; (b) multi-stage microfluidic platform that can connect up to six chip reactors in series ${ }^{[101]}$

学” 概念结合微反应器, 用于有机合成中危险中间体的 处理(图 4(a)). 微流控系统对反应过程有较高的控制力, 因此能有效控制危险中间产物的生成, 避免其大量咜 存. 通过对通道进行适当的设计, 能够以边生产边迅速 反应的方式缩短中间体的停留时间，降低了实验人员面 临的潜在风险, 使有机材料的合成更加绿色、环 保 ${ }^{[109-111]}$. 该技术现已得到进一步发展, Kim 和 Yoshida 等[112]将芯片中的混合区域设计为特殊的 3D 蛇形通道, 可以通过亚毫秒级的快速混合 “超越” 不希望发生的 弗里斯重排反应, 在该反应发生之前得到目标产物(图 4(b)). 由此可见, 微流控技术的独特优势使其有潜力完 成在常规反应器中难以实现的反应.

在传统反应容器中, 根据朗伯比尔定律, 光能穿透 的溶液深度是有限的, 光强度会随着穿透深度的增加而 呈现指数衰减, 容器内的光催化剂仅能得到部分活 化 ${ }^{[113]}$. 因此, 当使用常规反应器时, 光催化有机合成实 际上都是在 “光子受限” 的情况下进行的. 在微反应器 中，微米尺度的通道使得光穿透的深度短且分布均匀， 微反应器比表面积大的特点显著提高了光照面积, 在光 催化有机合成中具有天然的优势 [114-116]. 在最常见的光 催化有机合成微反应器中, 研究人员们选用内径数百微 米的毛细管，制成反应盘管，置于光照之下. 因此，光

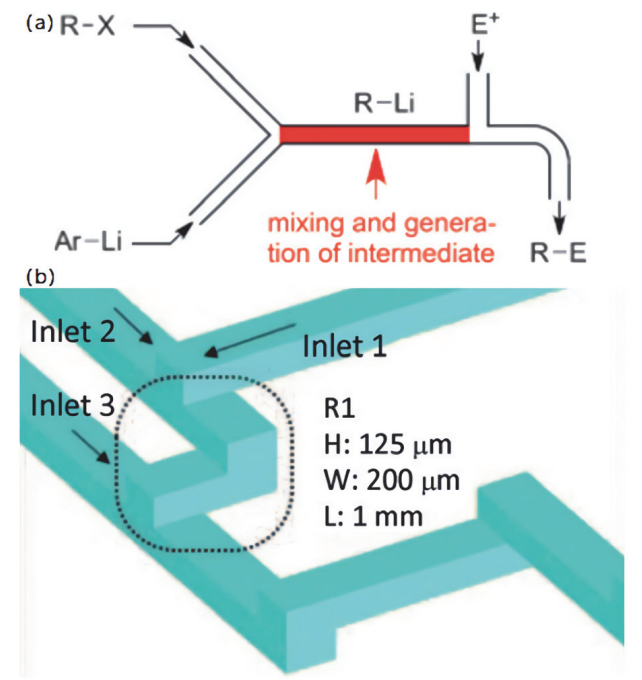

图 4 通过对通道的设计缩短中间体的寿命. (a) 缩短中间体的停留时 间 ${ }^{[108]}$; (b) 3D 蛇形通道混合区 ${ }^{[12]}$

Figure 4 Shorten the life of the intermediate through the design of the channel. (a) Shorten the residence time of the intermediate ${ }^{[108]}$; (b) mixed area with $3 \mathrm{D}$ serpentine channel ${ }^{[112]}$

催化有机合成及反应条件篎选的速率得到了明显的提 高. Laudadio 等 ${ }^{[117]}$ 除了利用上述光反应器之外, 还借助 微反应器能耐受高压的特点, 通过高压将气体液化, 避 免了气-液反应的传质限制，高选择性地完成了 $\mathrm{C}\left(\mathrm{sp}^{3}\right)-$ $\mathrm{H}$ 键的选择功能性活化. Perera 等 ${ }^{[118]}$ 开发的辉瑞流动化 学合成系统可在不同溶剂、温度、压力等条件下进行自 动化的高通量化学反应篮选. 自动进样器可从多达 192 个样品瓶中进行取样, 在 $45 \mathrm{~s}$ 内完成 2 个反应物、催化 剂、配体和碱 5 个组分的进样. 研究人员在 $4 \mathrm{~d}$ 内利用 该系统对 Suzuki-Miyaura 偶联反应进行了 5760 个条件 的篎选, 每天可篮选的条件数量高达 1500 个.

电化学有机合成一般选择微流控芯片反应器, 并在 芯片上加工符合要求的微电极. Mo 等 ${ }^{[119]}$ 加工了间隔 1 $\mu \mathrm{m}$ 的互相交叉的叉指电极(IDE), 在该芯片反应器上进 行自由基-自由基交叉偶联: $\alpha$-氨基 $\mathrm{C}-\mathrm{H}$ 芳基化反应.

常规有机材料的合成相当耗时, 反应时间通常需要 数分钟至数小时. 因此, 为了满足应用的要求, 亟需以 受控的方式加速有机材料的合成. 在对合成反应体系进 行合理设计的前提下, 微流控技术能快速且高效地合成 所需的有机材料, 为有机材料的合成提供更好的选择性 和更高的产率. 目前, 微流控技术已被应用于纤维、多 肽、壳聚糖和脂类等多种不同的有机小分子和高分子材 料的合成中 ${ }^{[120-126] . ~}$

\section{3 复合微/纳米材料}

复合材料由两种及以上的组分或材料组成，其特性 不仅仅是单个成分特性的简单加和, 还可产生更有利的 结果, 在包括医学诊断、建筑装饰和光子器件等领域都 有应用 ${ }^{[127]}$. 微流控技术具有的多步合成能力使其能对 颗粒形状、尺度、化学各向异性、孔隙率和核/壳结构 
等性质进行精细控制, 满足复合微/纳米材料集成多种 物理化学特性的需求 ${ }^{[128]}$. 迄今为止, 通过微流控技术 已经成功生产了有机材料基、金属基、无机非金属基等 多种复合材料[129-134], 其中比较有代表性的有 Janus 微 粒和金属有机骨架化合物.

Janus 微粒是一种由两个具有不同理化性质的部分 组成的典型复合材料. 其合成过程对反应条件的要求较 高, 通过常规方法进行合成难度较大 ${ }^{[135]}$. Min 等 ${ }^{[136]}$ 通 过微管路系统将聚苯乙烯(PS)和聚乳酸(PLA)两种聚合 物的混合物分散在甲苯中并形成液滴, 随后对液滴进行 溶剂蒸发和相分离等操作, 制备了包含 PS 和 PLA 的 Janus 颗粒(图 5). 此外, 微流控技术还被用于合成多种 形状可控的高质量 Janus 微粒 ${ }^{[137-139]}$.

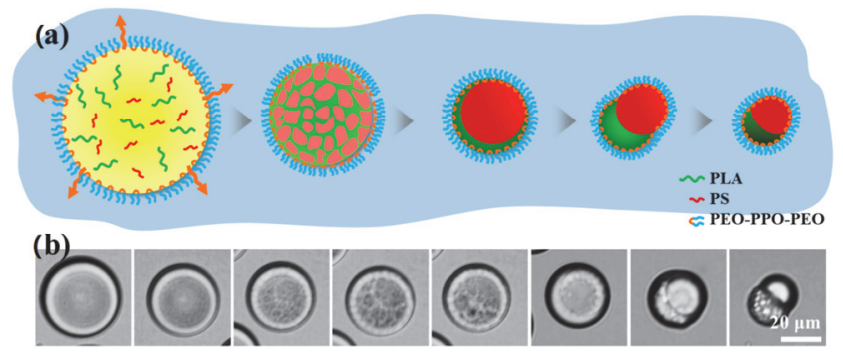

图 $5(a, b)$ 通过固结分散在聚环氧乙烷-聚环氧丙烷三嵌段共聚物水 溶液中的 PS 和 PLA 的甲苯乳液滴形成橡子状 Janus 微粒 ${ }^{[136]}$

Figure $5(a, b)$ Aqueous Janus microparticles formed by consolidation of toluene emulsion drops of PS and PLA dispersed in an aqueous polyethylene oxide-polypropylene oxide triblock copolymer solution ${ }^{[136]}$

金属有机骨架化合物 (metal organic frameworks, $\mathrm{MOFs}$ ) 是一类有机配体与无机金属中心通过自组装连 接的多功能复合多孔材料 ${ }^{[140]}$, 可应用于气体存储和分 离、生物医学、传感、非线性光学和能量转换等领 域 ${ }^{[141-144]}$. Faustini 等 ${ }^{[145]}$ 利用微流控系统在数分钟内成 功合成稳定性和催化性能更好的 HKUST-1、MOF-5 和 $\mathrm{UiO}-66$ 等代表性的均相 $\mathrm{MOFs}$ 材料, 以及 $\mathrm{Co}_{3} \mathrm{BTC}_{2} @$ $\mathrm{Ni}_{3} \mathrm{BTC}_{2} 、 \mathrm{MOF}-5 @ \mathrm{diCH}_{3}-\mathrm{MOF}-5$ 和 $\mathrm{Fe}_{3} \mathrm{O}_{4} @ \mathrm{ZIF}-8$ 几种 核-壳 MOFs 材料(图 6(a)). 与常规的水热法和溶剂热合 成法相比, 基于微流控技术合成的 MOFs 材料的形貌更 均匀 ${ }^{[146-147]}$. 此外, 微流控技术还可以通过构造均匀的 分级孔隙达到改善 MOFs 性能的目的(图 6(b) $)^{[148]}$.

\section{4 未来微流控技术在微/纳米材料合成领域的 主要发展趋势}

综上所述，微流控技术在微/纳米材料合成方面的 研究已经取得了显著的进展, 并且在充分利用微流控技 术的优势推动微/纳米材料合成的进步方面仍有着极大 的拓展空间和潜力. 以下将对该领域未来的主要发展趋 势进行介绍.
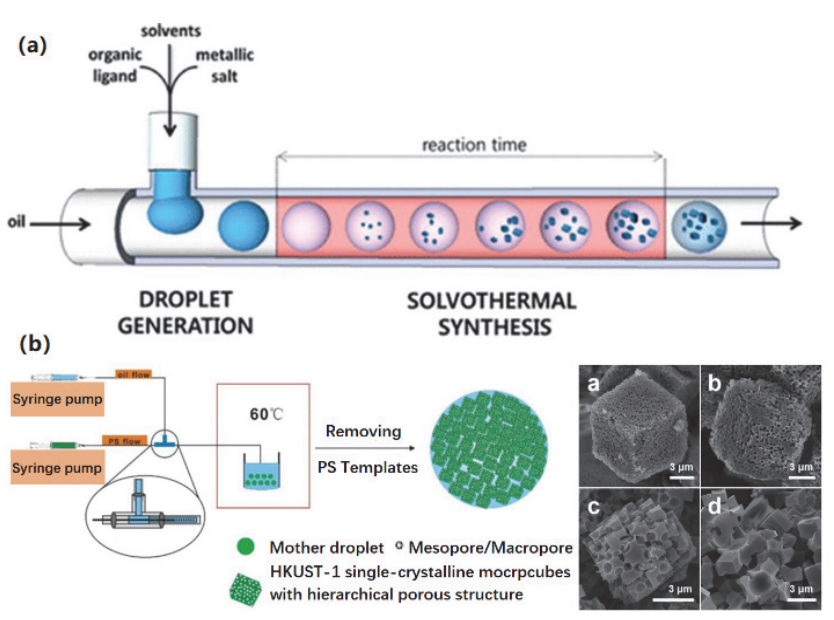

图 6 (a) 液滴微流控系统合成 MOFs 材料示意图 ${ }^{[145]}$; (b) 利用微流控 系统制备有着均匀的分级孔隙的 MOFs 材料 ${ }^{1148]}$

Figure 6 (a) Schematic illustration of MOFs synthesized by droplet microfluidic system ${ }^{[145]}$; (b) preparation of MOFs with uniform graded pores by microfluidic system ${ }^{[148]}$

\section{1 微流控技术与多种在线检测技术的联用}

常规有机合成中的产物检测通常是脱线进行的, 存 在效率低、耗样量大、费时费力且成本较高等问题. 通 过与多种在线检测技术联用，微流控技术能实时监控合 成反应，高效地获取材料的粒度、形状、组成和形态等 信息，并能根据反馈及时调整反应体积等条件，有望实 现对反应条件的高通量篮选.

与其它在线检测技术相比，苂光光谱、吸收光谱等 光学检测技术与微流控技术的联用最为常见 (图 $7(\mathrm{a}))^{[149]}$. 此外, 刘娇等 [150] 以磁性核壳纳米粒子 $\mathrm{Fe}_{3} \mathrm{O}_{4} @ \mathrm{Ag}$ 为表面增强拉曼光谱(SERS)基底, 使用微流 控反应器, 通过外加磁场调控富集实现了 $\alpha$-苯乙醇的微 量合成反应以及实时 SERS 监测, 结果表明微流控技术 与 SERS 联用可发展成为监测微量有机反应的有效手 段. 相比之下，电化学、色谱和质谱等其它检测技术与 微流控技术的联用仍比较少见 ${ }^{[151]}$. 这是由多种原因导 致的, 例如, 这些检测技术对样品的状态有特殊要求、 联用操作较为繁琐复杂、对反应器和检测器的接口有特 殊要求等. 但近年来也出现了相关研究, 例如, 夏海伦 等 ${ }^{[152]}$ 制备了可用于高真空环境中的微流控电化学池, 并且实现了液体电化学飞行时间 -二次离子质谱 (TOF-SIMS)联用, 将其应用于氯化钾溶液在不同电极 电位条件下迁移过程的实时监测.

微流控技术与在线检测技术的联用使得构建集合 成、检测、实时反馈及优化于一体的多功能系统的设想 成为可能(图 7(b) $)^{[153]}$. 此外, 这一设想还需要计算机的 强大的数据处理能力以及人工智能等技术作为支持. 随 着科技的发展，微型化、集成化与便携化已成为分析仪 器发展的主要方向. 对应用于材料合成与篮选的微流控 技术而言，与更加高效、全面、精确的在线检测技术联 用是重要的发展趋势之一. 


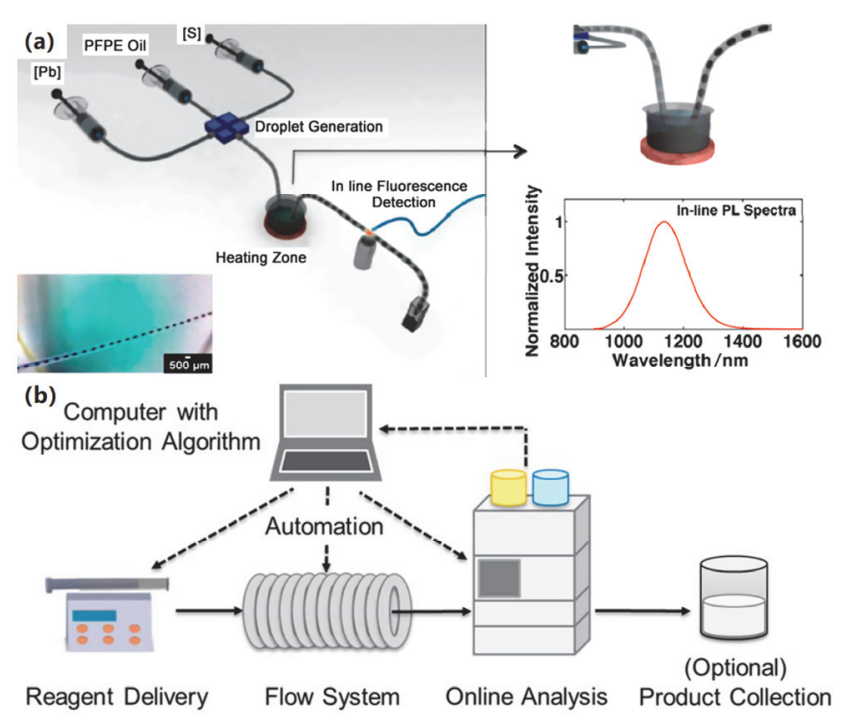

图 7 (a) 集成了在线 NIR 荧光检测系统的微反应器示意图 ${ }^{[149]}$; (b) 使用微流控系统进行自动反馈优化和动力学数据提取的工作流程示 意图 ${ }^{[153]}$

Figure 7 (a) Schematic of a microreactor integrated with an online NIR fluorescence detection system ${ }^{[149]}$; (b) schematic workflow for automatic feedback optimization and kinetic data extraction using a microfluidic system $^{[153]}$

\section{2 利用微流控技术实现微/纳米材料的多步骤复杂 合成及处理}

随着对微/纳米材料的功能化需求日益增加, 对反 应条件提出的要求也越来越复杂和严格, 比如需要表面 改性、修饰等. 经过合理设计, 微流控技术可以满足微/ 纳米材料多步骤复杂合成的需求 ${ }^{[154]}$. 目前, 微流控技 术已经可以通过对流路的设计实现样品的快速混合或 对微流体的操控等. 此外, 有些材料具有相对复杂的形 态或结构, 就会对系统提出更为严苛的要求, 希望能够 在合成过程中精确地控制反应条件和参数. 微流控系统 通过集成相应的部件可以实现对电、磁、光、温度等反 应条件的良好控制, 促进了这类功能性材料的发展. 在 近几年发展的系统中, 研究人员们充分展示了模块化方 法高灵活性的优势, 通过将样品(包括固体和液体样品) 的处理以及后续的加热、冷却、光照、混合等功能分别 集成在芯片上，构建了即插即用的模块化合成装置.

此外, 微/纳米材料的合成多数都需要根据具体要 求进行前处理(混合、溶解等)和后处理(过滤、纯化 等 ${ }^{[155-156]}$. 然而, 目前常用的微反应器仍存在难以处理 固体物质以及功能相对简单等问题, 尚不能满足复杂的 前处理和后处理的需求.

对于流动反应器而言, 如何进行涉及固体物质的反 应一直是一个具有普遍性的难点. 若反应中存在不溶或 难溶的固体物质, 很可能导致注射器堵塞、通道结垢、 进样困难、切换阀损坏等严重的问题. 目前, 绝大多数 流动反应都会选择溶解性较好的物质参与反应, 尽量避 免难溶或不溶物质的引入.
在这一点上, 间歇反应器对固体物质的包容性高于 流动反应器. 但是, 无论是间歇还是流动反应器, 在反 应的前处理和后处理部分都依然面临着固体物质的处 理难题. 例如, 过滤与纯化是研究人员最常用的涉及固 体物质的后处理操作, 是进行气相色谱、液相色谱和核 磁波谱等检测之前必不可少的步骤. 然而, 这些操作目 前绝大多数依然需要依靠人工来完成, 不易实现自动化 和在线化. 固体物质的处理难以集成化和自动化的问题 已经严重影响了检测方法的在线集成，对反应条件篮选 的高效率以及系统的通用性都提出了挑战.

\section{3 基于微流控技术将微/纳米材料合成的规模扩大 至工业水平}

如前所述, 研究人员们利用微流控技术已经合成出 多种高质量的微/纳米材料. 然而, 对于这些微/纳米材 料而言，如何将合成规模扩大至工业水平是其从实验室 走向具体应用的过程中必须要解决的问题 ${ }^{[157]}$. 目前在 实验室中搭建的微流控系统多数只能实现微/纳米材料 在克级产量的小规模合成，在大规模生产中仍面临着很 大挑战. 其中原因之一是单纯增加微反应器的通道体积 通常会导致传质、传热及混合效率降低，对反应条件的 控制力随之下降, 继而难以保证最终产物的质量及产 率.

2014 年, 骆广生等 ${ }^{[158]}$ 在解释 “微化工技术” 时也 提到了扩大合成规模的问题. “微化工技术” 是一种以 微结构元件为核心，在微米或亚毫米的受限空间内进行 的化工过程. 该技术以 “数量放大” 为基本准则, 将实 验室中的微设备快速扩展并运用于工业过程中, 具体方 法是通过平行设置多个相同的反应通道成倍提高反应 通量, 同时无需更改反应器的几何形状. 因此, 该技术 能够在不对基础化学过程进行任何修改的前提下增加 通量, 理论上可以既不降低产品的质量, 又达到扩大规 模的目的. 此外, Nightingale 等 ${ }^{[159]}$ 利用多通道液滴反应 器, 在 $9 \mathrm{~h}$ 内得到了 $54.4 \mathrm{~g}$ 纯化后的 CdTe 晶体, 相当于 每天 $145 \mathrm{~g}$ 的产量. 该反应器的结构具有可扩展性, 只 需进行最少的修改, 就能够达到工业上希望的每天公斤 级的生产水平. 该方法面临的主要挑战是如何保证各通 道产品的一致性, 这要求该微流控系统具有良好的稳定 性，并确保各通道之间的一致性.

此外, 市场上也出现了商品化的流动合成仪器. 英 国的 Vapourtec 流动合成仪具有完成较大规模合成的潜 力. 荷兰的 Chemtrix 公司不仅开发了可实现反应篮选的 毫克级化学合成反应器, 还研发了适合工业应用的吨级 化学合成反应器(例如 Plantrix MR555 流动式反应器), 为具有挑战性的化学药品的大规模合成提供了选择.

\section{4 发展适合高通量合成与篎选的新的微流体驱动和 控制方法}

如前所述，微流控技术在高通量化学合成与篎选中 具有独特的优势和重要的潜力. 高通量合成与篮选往往 
涉及多个组分, 并且需要改变参与反应的物质种类以及 各组分之间的配比等条件. 然而, 现有微流控合成系统 中所采用的流体驱动方式(例如: 注射洜、蠕动洜和气压 泵等), 都各自存在不同的优势和局限性. 例如: (a)注射 洜对流量的控制比较稳定, 但存在着换液困难、不易混 合均匀的问题. (b)蠕动洜是非接触式洜, 但在涉及有机 反应时，泵管的选择受限. 对有机溶剂耐受性好的 Teflon 管存在弹性较差的问题, 会因为磨损而需要经常 更换, 因此如何保持稳定的流量成了一个难题. (c)气压 洜在保证无水无氧的反应条件方面具有优势, 但由于不 同液体的粘度不同, 对流量进行稳定和精确的控制较为 困难.

此外，高通量合成与篮选除了要求系统能够快速、 高效地完成样品的选择和配置之外, 还需要进样方法能 够避免不必要的交叉污染, 并保证样品混合均匀. 这些 都需要创新发展新的微流体驱动和控制方法.

\section{5 间歇和流动反应器的结合使用}

如上文所述, 间歇反应器与流动反应器各有其独特 的优势, 也存在各自的问题. 例如: (a)间歇反应器更易 于通过多个反应器并行实现高通量, 也不容易存在交叉 污染的问题, 易于集成加热、搅拌、光照等设施; 但在 系统放大时容易遇到重复性较差的问题, 且难以满足工 业化生产的需求. (b)流动反应器在传质、传热性能上具 有优势, 可提高合成反应的效率, 并且更易于扩大至工 业生产需要的水平. 但在进行化学合成条件的筱选时, 流动反应器在篮选条件的通量和反应种类方面受到较 大限制。

因此, 将二者结合起来, 各取所长, 取长补短, 将 是该领域今后重要的发展方向之一. 这一目标的实现, 将使微流控技术在化学合成中的实用性和普适性得到 进一步的提高.

\section{6 结合人工智能技术, 实现智能化合成与篎选}

微流控技术在高通量反应条件篮选方面具有得天 独厚的优势. 在反应和检测的速率都获得明显提高的前 提下, 数据处理和分析的速度和深度将会成为实现进一 步高通量合成与笁选的限制因素. 因此, 未来微流控技 术将与人工智能技术结合, 以显著提高数据处理和分析 的速度和深度, 不仅能对实验结果进行及时的分析及反 馈, 还能从中提炼出有用的信息, 为下一步优化提供建 议, 从而自动化地完成反应条件的循环迭代篮选, 实现 “化学合成科研机器人”的目标. 这样高效的工具能够代 替研究人员完成费时费力的实验条件优化的工作, 将研 究人员从这种重复的工作中解放出来, 使他们可以将宝 贵的时间和精力花在更加有意义和创新性的地方.

另外, 由于人工智能技术具有通过深度学习处理海 量数据的能力, 使其有望分析一些复杂程度更高或是目 前人类尚且难以预测的反应, 帮助研究人员开拓思路,
进一步拓展合成反应更多的可能性.

\section{5 总结与展望}

近年来，与传统的合成方法相比，微流控技术因其 独特的优势逐渐吸引了研究者们的目光，作为一项新兴 的技术在材料合成领域取得了快速发展，成为高质量 微/纳米材料合成的一种新选择.

本综述介绍了微流控技术应用在微/纳米材料合成 领域的独特优势, 从反应器的结构形式和反应方式两个 角度分别综述了应用于微/纳米材料合成的多种常见微 反应器. 此外, 本综述从无机材料、有机材料和复合材 料的合成方面介绍了微流控技术在微/纳米材料合成领 域的具体应用，并展望了未来该领域的主要发展趋势. 尽管通过微流控技术已经成功得到了许多引人注目的 研究成果, 但是该领域在联用在线检测技术、多步骤复 杂合成及处理、产量合成规模扩大化、固体物质的处理、 新驱动方式的发展以及间歇与流动反应器结合等方面 依然具有很多潜力有待进一步发掘.

总而言之，微流控技术在微/纳米材料的合成领域 有着广泛的适用性以及良好的发展前景. 微流控技术为 微/纳米材料的合成提供了新的思路和方法，在工业生 产和学术研究中都蕴含着丰富的可能性和巨大的潜力.

\section{作者简介}

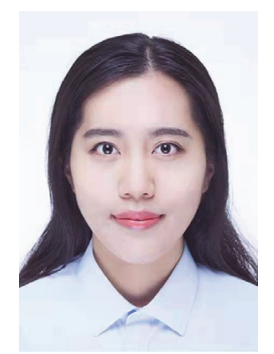

卢佳敏, 浙江大学化学系博士研究生. 2019 年于厦门大学 获得学士学位, 2019 年起在方群教授的指导下于浙江大学攻 读博士学位, 目前研究方向主要包括微流控分析、流动化学与 材料合成.

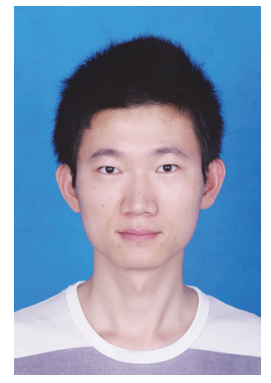

王慧峰, 浙江大学杭州国际科创中心博士后研究员. 2020 年在浙江大学获得分析化学方向理学博士学位. 目前研究方 向主要包括微流控分析、流动化学与单细胞分析. 


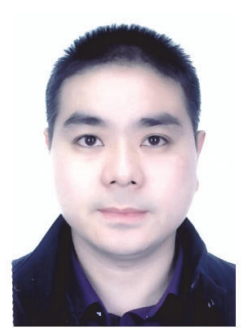

潘建章, 副研究员, 硕士生导师, 致力于微流控分析仪器 的微型化、自动化、智能化研究工作. 成功研制掌上激光诱导 荧光-毛细管电泳生化分析仪、手持超微量液芯波导光度计、 面向航空航天应用的高速毛细管电泳仪、PET 显像剂全自动 合成仪等微型化自动化微流控仪器. 已发表 SCI 论文 20 余篇, 获授权国家发明专利 7 项, 主持和参与包括国家自然科学基 金面上项目、科学仪器研制专项、重大科研仪器研制项目等 10 余项.

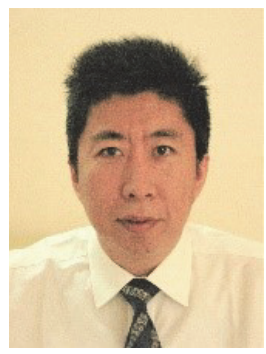

方群, 浙江大学求是特聘教授, 博士生导师, 化学系微分 析系统研究所所长, 国家杰出青年基金获得者. 1998 年在沈阳 药科大学获得药物分析学博士学位. 自 1998 年开始从事微流 控分析的研究工作. 目前研究方向包括微流控液滴分析, 微 流控毛细管电泳、液相色谱和质谱分析, 微型化分析系统研制, 以及微流控系统在单细胞分析、微量生化分析、药物篮选和 现场分析中的应用. 发表研究论文 130 余篇. 在微流控分析领 域申请国家发明专利 40 项, 其中 26 项获得授权. 曾主持承担 国家基金委重大项目课题、国家杰出青年基金、国家基金重 点项目、科学仪器研制专项和面上项目等.

\section{References}

[1] Deng, Y.; Liu, K. K.; Liu, Y.; Dong, H. M.; Li, S. J. Nanosci. Nanotechnol. 2016, 16, 9460.

[2] Yang, P. Q.; Peng, J. M.; Chu, Z. Y.; Jiang, D. F.; Jin, W. Q. Biosens. Bioelectron. 2017, 92, 709 .

[3] Yue, Z.; Lisdat, F.; Parak, W. J.; Hickey, S. G.; Tu, L. P.; Sabir, N.; Dorfs, D.; Bigall, N. C. ACS Appl. Mater. Interfaces 2013, 5, 2800.

[4] Cao, X. H.; Tan, C. L.; Sindoro, M.; Zhang, H. Chem. Soc. Rev. 2017, 46, 2660.

[5] Cao, X. H.; Tan, C. L.; Zhang, X.; Zhao, W.; Zhang, H. Adv. Mater. 2016, 28, 6167.

[6] Pan, X. L.; Hong, X. F.; Xu, L.; Li, Y. X.; Yan, M. Y.; Mai, L. Q. Nano Today 2019, 28, 100764

[7] Shen, H. B.; Gao, Q.; Zhang, Y. B.; Lin, Y.; Lin, Q. L.; Li, Z. H.; Chen, L.; Zeng, Z. P.; Li, X. G.; Jia, Y.; Wang, S. J.; Du, Z. L.; Li, L. S.; Zhang, Z. Y. Nat. Photonics 2019, 13, 192.

[8] Yuan, F. L.; Yuan, T.; Sui, L. Z.; Wang, Z. B.; Xi, Z. F.; Li, Y. C.; Li, X. H.; Fan, L. Z.; Tan, Z. A; Chen, A. M.; Jin, M. X.; Yang, S. H. Nat. Commun. 2018, 9, 2249.

[9] Zhao, M. X.; Zhu, B. J. Nanoscale Res. Lett. 2016, 11, 207.

[10] Faria, M.; Bjornmalm, M.; Thurecht, K. J.; Kent, S. J.; Parton, R. G.; Kavallaris, M.; Johnston, A. P. R.; Gooding, J. J.; Corrie, S. R.; Boyd, B. J.; Thordarson, P.; Whittaker, A. K.; Stevens, M. M.; Prestidge, C. A.; Porter, C. J. H.; Parak, W. J.; Davis, T. P.; Crampin, E.
J.; Caruso, F. Nat. Nanotechnol. 2018, 13, 777.

[11] Yang, G. B.; Xu, L. G.; Chao, Y.; Xu, J.; Sun, X. Q.; Wu, Y. F.; Peng, R.; Liu, Z. Nat. Commun. 2017, 8, 902.

[12] Ma, X.; Feng, H. H.; Liang, C. Y.; Liu, X. J.; Zeng, F. Y.; Wang, Y. J. Mater. Sci. Technol. 2017, 33, 1067.

[13] Zhang, L. J.; Xia, L.; Xie, H. Y.; Zhang, Z. L.; Pang, D. W. Anal. Chem. 2019, 91, 532 .

[14] Rosa, L.; Blackledge, J.; Boretti, A. Biomedicines 2017, 5, 7.

[15] Prieto, G.; Tueysuez, H.; Duyckaerts, N.; Knossalla, J.; Wang, G. H.; Schueth, F. Chem. Rev. 2016, 116, 14056.

[16] Zhu, Q. L.; Xia, W.; Akita, T.; Zou, R. Q.; Xu, Q. Adv. Mater. 2016, $28,6391$.

[17] Lignos, I.; Maceiczyk, R.; deMello, A. J. Acc. Chem. Res. 2017, 50 , 1248.

[18] Protiere, M.; Nerambourg, N.; Renard, O.; Reiss, P. Nanoscale Res. Lett. 2011, 6, 472.

[19] Liu, Y.; Jiang, X. Lab Chip 2017, 17, 3960.

[20] Boken, J.; Soni, S. K.; Kumar, D. Crit. Rev. Anal. Chem. 2016, 46 , 538.

[21] Xu, D. D.; Huang, X. W.; Guo, J. H.; Ma, X. Biosens. Bioelectron. 2018, 110,78 .

[22] Bae, S.; Han, H.; Bae, J. G.; Lee, E. Y.; Im, S. H.; Kim, D. H.; Seo, T. S. Small 2017, 13, 1603392.

[23] Liu, W. W.; Zhu, Y.; Fang, Q. Anal. Chem. 2017, 89, 6678.

[24] Li, Z. Y.; Huang, M.; Wang, X. K.; Zhu, Y.; Li, J. S.; Wong, C. C. L.; Fang, Q. Anal. Chem. 2018, 90, 5430.

[25] Zhu, Y.; Zhu, L. N.; Guo, R.; Cui, H. J.; Ye, S.; Fang, Q. Sci. Rep. 2014, 4, 5046.

[26] Du, X. H.; Li, W. M.; Du, G. S.; Cho, H.; Yu, M.; Fang, Q.; Lee, L. P.; Fang, J. Anal. Chem. 2018, 90, 3253.

[27] Fang, H. B.; Sun, S. J.; Liao, P. S.; Hu, Y.; Zhang, J. Y. J. Mater. Chem. A 2018, 6, 2115.

[28] Wang, Q.; Steinbock, P. O. ChemCatChem 2020, 12, 63.

[29] Capretto, L.; Carugo, D.; Mazzitelli, S.; Nastruzzi, C.; Zhang, X. L. Adv. Drug Delivery Rev. 2013, 65, 1496.

[30] Huang, Q. S.; Mao, S. F.; Khan, M.; Lin, J. M. Analyst 2019, 144, 808.

[31] Xu, H.; Gao, M. X.; Tang, X. Q.; Zhang, W. Q.; Luo, D.; Chen, M. Small Methods 2020, 4, 1900506.

[32] Ran, R.; Sun, Q.; Baby, T.; Wibowo, D.; Middelberg, A. P. J.; Zhao, C. X. Chem. Eng. Sci. 2017, 169, 78.

[33] Shembekar, N.; Chaipan, C.; Utharala, R.; Merten, C. A. Lab Chip 2016, 16, 1314 .

[34] Yue, J. Catal. Today 2018, 308, 3.

[35] Edel, J. B.; Fortt, R.; deMello, J. C.; deMello, A. J. Chem. Commun. 2002, 1136 .

[36] Akdas, T.; Haderlein, M.; Walter, J.; Zubiri, B. A.; Spiecker, E.; Peukert, W. RSC Adv. 2017, 7, 10057.

[37] Li, G. X.; Pu, X.; Shang, M. J.; Zha, L.; Su, Y. H. AIChE J. 2019, 65, 334.

[38] deMello, A. J. Nature 2006, 442, 394

[39] Zhang, Y. S.; Aleman, J.; Shin, S. R.; Kilic, T.; Kim, D.; Shaegh, S. A. M.; Massa, S.; Riahi, R.; Chae, S.; Hu, N.; Avci, H.; Zhang, W. J.; Silvestri, A.; Nezhad, A. S.; Manbohi, A.; De Ferrari, F.; Polini, A.; Calzone, G.; Shaikh, N.; Alerasool, P.; Budina, E.; Kang, J.; Bhise, N.; Ribas, J.; Pourmand, A.; Skardal, A.; Shupe, T.; Bishop, C. E.; Dokmeci, M. R.; Atala, A.; Khademhosseini, A. Proc. Natl. Acad. Sci. U. S. A. 2017, 114, E2293.

[40] Lu, M.; Ozcelik, A.; Grigsby, C. L.; Zhao, Y.; Guo, F.; Leong, K. W.; Huang, T. J. Nano Today 2016, 11, 778.

[41] Elvira, K. S.; Solvas, X. C. i.; Wootton, R. C.; deMello, A. J. Nat. Chem. 2013, 5, 905

[42] Fanelli, F.; Parisi, G.; Degennaro, L.; Luisi, R. Beilstein J. Org. Chem. 2017, 13, 520 .

[43] Malet-Sanz, L.; Susanne, F. J. Med. Chem. 2012, 55, 4062.

[44] Abdel-Latif, K.; Epps, R. W.; Kerr, C. B.; Papa, C. M.; Castellano, F. N.; Abolhasani, M. Adv. Funct. Mater. 2019, 29, 1900712.

[45] Epps, R. W.; Felton, K. C.; Coley, C. W.; Abolhasani, M. Lab Chip 2017, 17, 4040

[46] Bian, F.; Wang, H.; Sun, L. Y.; Liu, Y. X.; Zhao, Y. J. J. Mater Chem. B 2018, 6, 7257.

[47] Kumar, S.; Ganesan, S. Comput. Chem. Eng. 2017, 96, 128.

[48] Maceiczyk, R. M.; Bezinge, L.; deMello, A. J. React. Chem. Eng. 2016, $1,261$.

[49] Lazzari, S.; Theiler, P. M.; Shen, Y.; Coley, C. W.; Stemmer, A.; Jensen, K. F. Langmuir 2018, 34, 3307. 
[50] Maceiczyk, R. M.; Lignos, I. G.; deMello, A. J. Curr. Opin. Chem. Eng. 2015, 8, 29.

[51] Liang, Y. R.; Zhu, L. N.; Gao, J.; Zhao, H. X.; Zhu, Y.; Ye, S.; Fang, Q. ACS Appl. Mater. Interfaces 2017, 9, 11837.

[52] Zhao, S. P.; Ma, Y.; Lou, Q.; Zhu, H.; Yang, B.; Fang, Q. Anal. Chem. 2017, 89, 10153.

[53] Pan, L. J.; Tu, J. W.; Ma, H. T.; Yang, Y. J.; Tian, Z. Q.; Pang, D. W.; Zhang, Z. L. Lab Chip 2017, 18, 41.

[54] Zhang, L.; Chen, Q. H.; Ma, Y.; Sun, J. S. ACS Appl. Bio Mater. 2019, 3, 107.

[55] Kubendhiran, S.; Bao, Z.; Dave, K.; Liu, R. S. ACS Appl. Nano Mater. 2019, 2, 1773.

[56] Plutschack, M. B.; Pieber, B.; Gilmore, K.; Seeberger, P. H. Chem. Rev. 2017, 117, 11796.

[57] Chan, E. M.; Mathies, R. A.; Alivisatos, A. P. Nano Lett. 2003, 3, 199.

[58] Atobe, M.; Tateno, H.; Matsumura, Y. Chem. Rev. 2018, 118, 4541.

[59] Nightingale, A. M.; deMello, J. C. Adv. Mater. 2013, 25, 1813.

[60] Yen, B. K. H.; Stott, N. E.; Jensen, K. F.; Bawendi, M. G. Adv. Mater. 2003, 15, 1858

[61] Bedard, A.-C.; Adamo, A.; Aroh, K. C.; Russell, M. G.; Bedermann, A. A.; Torosian, J.; Yue, B.; Jensen, K. F.; Jamison, T. F. Science 2018, 361, 1220 .

[62] Swyer, I.; Soong, R.; Dryden, M. D. M.; Fey, M.; Maas, W. E.; Simpson, A.; Wheeler, A. R. Lab Chip 2016, 16, 4424.

[63] Swyer, I.; von der Ecken, S.; Wu, B.; Jenne, A.; Soong, R.; Vincent, F.; Schmidig, D.; Frei, T.; Busse, F.; Stronks, H. J.; Simpson, A. J.; Wheeler, A. R. Lab Chip 2019, 19, 641.

[64] Wu, B.; von der Ecken, S.; Swyer, I.; Li, C. L.; Jenne, A.; Vincent, F.; Schmidig, D.; Kuehn, T.; Beck, A.; Busse, F.; Stronks, H.; Soong, R.; Wheeler, A. R.; Simpson, A. Angew. Chem., Int. Ed. 2019, 58, 15372.

[65] Li, G.; Parmar, M.; Lee, D. W. Lab Chip 2015, 15, 766.

[66] Santanilla, A. B.; Regalado, E. L.; Pereira, T.; Shevlin, M.; Bateman, K.; Campeau, L.-C.; Schneeweis, J.; Berritt, S.; Shi, Z. C.; Nantermet, P.; Liu, Y.; Helmy, R.; Welch, C. J.; Vachal, P.; Davies, I. W.; Cernak, T.; Dreher, S. D. Science 2015, 347, 49.

[67] Ahneman, D. T.; Estrada, J. G.; Lin, S.; Dreher, S. D.; Doyle, A. G. Science 2018, 360, 186.

[68] McNally, A.; Prier, C. K.; MacMillan, D. W. Science 2011, 334, 1114.

[69] Gemoets, H. P. L.; Su, Y. H.; Shang, M. J.; Hessel, V.; Luque, R.; Noel, T. Chem. Soc. Rev. 2016, 45, 83.

[70] Britton, J.; Raston, C. L. Chem. Soc. Rev. 2017, 46, 1250.

[71] Yen, B. K. H.; Günther, A.; Schmidt, M. A.; Jensen, K. F.; Bawendi, M. G. Angew. Chem., Int. Ed. 2005, 117, 5583.

[72] Akdas, T.; Haderlein, M.; Walter, J.; Apeleo Zubiri, B.; Spiecker, E.; Peukert, W. RSC Adv. 2017, 7, 10057.

[73] Kaminski, T. S.; Garstecki, P. Chem. Soc. Rev. 2017, 46, 6210.

[74] Abolhasani, M.; Jensen, K. F. Lab Chip 2016, 16, 2775.

[75] Coley, C. W.; Abolhasani, M.; Lin, H.; Jensen, K. F. Angew. Chem., Int. Ed. 2017, 56, 9847

[76] Hsieh, H. W.; Coley, C. W.; Baumgartner, L. M.; Jensen, K. F.; Robinson, R. I. Org. Process Res. Dev. 2018, 22, 542.

[77] Polyzos, A.; O'Brien, M.; Petersen, T. P.; Baxendale, I. R.; Ley, S. V. Angew. Chem., Int. Ed. 2011, 50, 1190.

[78] Cranwell, P. B.; O'Brien, M.; Browne, D. L.; Koos, P.; Polyzos, A.; Pena-Lopez, M.; Ley, S. V. Org. Biomol. Chem. 2012, 10, 5774.

[79] Browne, D. L.; O'Brien, M.; Koos, P.; Cranwell, P. B.; Polyzos, A.; Ley, S. V. Synlett 2012, 23, 1402.

[80] Brzozowski, M.; O'Brien, M.; Ley, S. V.; Polyzos, A. Acc. Chem. Res. 2015, 48, 349.

[81] Phan Huy, H.; Park, H.; Kim, D.-P. J. Am. Chem. Soc. 2011, 133, 14765.

[82] Shang, L. R.; Cheng, Y.; Zhao, Y. J. Chem. Rev. 2017, 117, 7964.

[83] Shestopalov, I.; Tice, J. D.; Ismagilov, R. F. Lab Chip 2004, 4, 316.

[84] Pan, L. J.; Tu, J. W.; Ma, H. T.; Yang, Y. J.; Tian, Z. Q.; Pang, D. W.; Zhang, Z. L. Lab Chip 2018, 18, 41.

[85] Liu, L. B.; Xiang, N.; Ni, Z. H. Electrophoresis 2020, 41, 833

[86] Le, N. H. A.; Van Phan, H.; Yu, J.; Chan, H. K.; Neild, A.; Alan, T. Int. J. Nanomed. 2018, 13, 1053.

[87] Chen, Y. G.; Wang, X.; He, X.; An, Q.; Zuo, Z. W. J. Am. Chem. Soc. 2021, 143, 4896.

[88] Sun, A. C.; Steyer, D. J.; Allen, A. R.; Payne, E. M.; Kennedy, R. T.; Stephenson, C. R. J. Nat. Commun. 2020, 11, 6202.
[89] Chiu, D. T.; deMello, A. J.; Di Carlo, D.; Doyle, P. S.; Hansen, C.; Maceiczyk, R. M.; Wootton, R. C. R. Chem 2017, 2, 201.

[90] Xu, L.; Peng, J. H.; Yan, M.; Zhang, D.; Shen, A. Q. Chem. Eng. Process. 2016, 102, 186.

[91] Navin, C. V.; Krishna, K. S.; Theegala, C. S.; Kumar, C. S. S. R. Nanotechnol. Rev. 2014, 3, 39.

[92] Watt, J.; Hance, B. G.; Anderson, R. S.; Huber, D. L. Chem. Mater. 2015, 27, 6442 .

[93] Ma, J.; Li, C. W. Sens. Actuators, B 2018, 262, 236.

[94] Gómez-Graña, S.; Fernández-López, C.; Polavarapu, L.; Salmon, J.-B.; Leng, J.; Pastoriza-Santos, I.; Pérez-Juste, J. Chem. Mater. 2015, 27, 8310.

[95] Sebastian, V.; Zaborenko, N.; Gu, L.; Jensen, K. F. Cryst. Growth Des. 2017, 17, 2700.

[96] Li, J. Z.; Wang, H. F.; Lin, L.; Fang, Q.; Peng, X. G. J. Am. Chem. Soc. 2018, 140, 5474.

[97] Guidelli, E. J.; Lignos, I.; Yoo, J. J.; Lusardi, M.; Bawendi, M. G.; Baffa, O.; Jensen, K. F. Chem. Mater. 2018, 30, 8562.

[98] Yang, H. W.; Luan, W. L.; Wan, Z.; Tu, S.-t.; Yuan, W. K.; Wang, Z. M. Cryst. Growth Des. 2009, 9, 4807.

[99] Bian, F. K.; Sun, L. Y.; Cai, L. J.; Wang, Y.; Zhao, Y. J. Wiley Interdiscip. Rev.: Nanomed. Nanobiotechnol. 2019, 133, 199.

[100] Lignos, I.; Stavrakis, S.; Nedelcu, G.; Protesescu, L.; deMello, A. J.; Kovalenko, M. V. Nano Lett. 2016, 16, 1869.

[101] Baek, J.; Shen, Y.; Lignos, I.; Bawendi, M. G.; Jensen, K. F. Angew. Chem., Int. Ed. 2018, 57, 10915.

[102] Xu, B. Y.; Yang, Z. Q.; Xu, J. J.; Xia, X. H.; Chen, H. Y. Chem. Commun. 2012, 48, 11635.

[103] Li, S. W.; Xu, H. H.; Wang, Y. J.; Luo, G. S. Langmuir 2008, 24, 4194.

[104] Baah, D.; Tigner, J.; Bean, K.; Britton, B.; Walker, N.; Henderson, G.; Floyd-Smith, T. Mater. Sci. Eng., B 2011, 176, 883.

[105] Jiao, M. X.; Zeng, J. F.; Jing, L. H.; Liu, C. Y.; Gao, M. Y. Chem. Mater. 2015, 27, 1299.

[106] Hao, R.; Deng, X.; Yang, Y. B.; Chen, D. Y. Acta Chim. Sinica 2014, 72, 1199 (in Chinese). (郝锐, 邓霄，杨毅彪，陈德勇，化学学报, 2014, 72, 1199.)

[107] Yoshida, J.-i. Chem. Commun. 2005, 4509.

[108] Yoshida, J.-i.; Nagaki, A.; Yamada, T. Chem.-Eur. J. 2008, 14, 7450.

[109] Yoshida, J.-i.; Kim, H.; Nagaki, A. ChemSusChem 2011, 4, 331.

[110] Wirth, T. Angew. Chem., Int. Ed. 2017, 56, 682.

[111] Bedard, A.-C.; Adamo, A.; Aroh, K. C.; Russell, M. G.; Bedermann, A. A.; Torosian, J.; Yue, B.; Jensen, K. F.; Jamison, T. F. Science 2018, 361, 1220 .

[112] Kim, H.; Min, K.-I.; Inoue, K.; Im, D. J.; Kim, D.-P.; Yoshida, J.-i Science 2016, 352, 691.

[113] Liu, A. L.; Li, Z. Q.; Wu, Z. Q.; Xia, X. H. Talanta 2018, 182, 544.

[114] Lin, C. H.; Chen, Y.; Su, Y. A.; Luo, Y. T.; Shih, T. T.; Sun, Y. C. Anal. Chem. 2017, 89, 5891.

[115] Jang, S.; Vidyacharan, S.; Ramanjaneyulu, B. T.; Gyak, K.-W.; Kim, D.-P. React. Chem. Eng. 2019, 4, 1466.

[116] Suhadolnik, L.; Pohar, A.; Likozar, B.; Ceh, M. Chem. Eng. J. 2016 303, 292.

[117] Laudadio, G.; Deng, Y.; van der Wal, K.; Ravelli, D.; Nuno, M.; Fagnoni, M.; Guthrie, D.; Sun, Y. H.; Noel, T. Science 2020, 369, 92

[118] Perera, D.; Tucker, J. W.; Brahmbhatt, S.; Helal, C. J.; Chong, A.; Farrell, W.; Richardson, P.; Sach, N. W. Science 2018, 359, 429.

[119] Mo, Y. M.; Rughoobur, G.; Nambiar, A. M. K.; Zhang, K.; Jensen, K. F. Angew. Chem., Int. Ed. 2020, 47, 20890.

[120] Persson, N. E.; Chu, P. H.; McBride, M.; Grover, M.; Reichmanis, E. Acc. Chem. Res. 2017, 50, 932.

[121] Arnon, Z. A.; Vitalis, A.; Levin, A.; Michaels, T. C. T.; Caflisch, A.; Knowles, T. P. J. Nat. Commun. 2016, 7, 13190.

[122] Yadavali, S.; Jeong, H.-H.; Lee, D.; Issadore, D. Nat. Commun. 2018, 9, 1222.

[123] Garg, S.; Heuck, G.; Ip, S.; Ramsay, E. J. Drug Targeting 2016, 24 , 821.

[124] Bell, R. V.; Parkins, C. C.; Young, R. A.; Preuss, C. M.; Stevens, M. M.; Bon, S. A. F. J. Mater. Chem. A 2016, 4, 813.

[125] Xu, J. H.; Zhao, H.; Lan, W. J.; Luo, G. S. Adv. Healthcare Mater 2012, 1,106 .

[126] Yang, C. H.; Huang, K. S.; Wang, C. Y.; Hsu, Y. Y. Electrophoresis 2012, 33, 3173

[127] Sarkar, J.; Ghosh, P.; Adil, A. Renewable Sustainable Energy Rev. 2015, 43, 164. 
[128] Dendukuri, D.; Doyle, P. S. Adv. Mater. 2009, 21, 4071.

[129] Ekanem, E. E.; Zhang, Z.; Vladisavljevic, G. T. J. Colloid Interface Sci. 2017, 498, 387.

[130] Li, Y. N.; Yan, D.; Fu, F. F.; Liu, Y. X.; Zhang, B.; Wang, J.; Shang, L. R.; Gu, Z. Z.; Zhao, Y. J. Sci. China Mater. 2017, 60, 543.

[131] Chen, L. F.; Yu, W.; Xie, H. Q. Powder Technol. 2012, 231, 18.

[132] Liang, F. X.; Zhang, C. L.; Yang, Z. Z. Adv. Mater. 2014, 26, 6944.

[133] Min, N. G.; Ku, M.; Yang, J.; Kim, S.-H. Chem. Mater. 2016, 28, 1430.

[134] Xu, Z. Q.; Yan, B.; Riordon, J.; Zhao, Y.; Sinton, D.; Moffitt, M. G. Chem. Mater. 2015, 27, 8094.

[135] Winkless, L. Mater. Today 2015, 18, 61.

[136] Min, N. G.; Kim, B.; Lee, T. Y.; Kim, D.; Lee, D. C.; Kim, S. H. Langmuir 2015, 31, 937.

[137] Hu, Y. D.; Wang, S. B.; Abbaspourrad, A.; Ardekani, A. M. Langmuir 2015, 31, 1885 .

[138] Ge, X. H.; Huang, J. P.; Xu, J. H.; Chen, J.; Luo, G. S. Soft Matter 2016, 12, 3425.

[139] Sun, X. T.; Guo, R.; Wang, D. N.; Wei, Y. Y.; Yang, C. G.; Xu, Z. R. J. Colloid Interface Sci. 2019, 553, 631.

[140] Wang, S. Z.; McGuirk, C. M.; d'Aquino, A.; Mason, J. A.; Mirkin, C. A. Adv. Mater. 2018, 30, 1800202.

[141] Rubio-Martinez, M.; Avci-Camur, C.; Thornton, A. W.; Imaz, I.; Maspoch, D.; Hill, M. R. Chem. Soc. Rev. 2017, 46, 3453.

[142] Jiao, L.; Wang, Y.; Jiang, H. L.; Xu, Q. Adv. Mater. 2018, 30, 1703663.

[143] Mahmood, A.; Guo, W.; Tabassum, H.; Zou, R. Adv. Energy Mater. 2016, 6, 1600423.

[144] Li, B.; Wen, H. M.; Cui, Y.; Zhou, W.; Qian, G.; Chen, B. Adv. Mater. 2016, 28,8819 .

[145] Faustini, M.; Kim, J.; Jeong, G. Y.; Kim, J. Y.; Moon, H. R. J. Am. Chem. Soc. 2013, 135, 14619.

[146] Bai, Y.; Dou, Y.; Xie, L. H.; Rutledge, W.; Li, J. R.; Zhou, H. C. Chem. Soc. Rev. 2016, 45, 2327.
[147] Wang, Y.; Li, L. J.; Yan, L. T.; Cao, L.; Dai, P. C.; Gu, X.; Zhao, X. B. Chin. Chem. Lett. 2018, 29, 849.

[148] Cui, J.; Gao, N.; Yin, X.; Zhang, W.; Liang, Y.; Tian, L.; Zhou, K. Nanoscale 2018, 10, 9192.

[149] Pu, Y.; Cai, F. H.; Wang, D.; Wang, J. X.; Chen, J. F. Ind. Eng. Chem. Res. 2018, 57, 1790.

[150] Liu, J.; Sun, H. L.; Yin, L.; Yuan, Y. X.; Xu, M. M.; Yao, J. L. Acta Chim. Sinica 2019, 77, 257 (in Chinese). (刘娇, 孙海龙, 印璐，袁 亚仙，徐敏敏，姚建林，化学学报, 2019, 77, 257.)

[151] Heiland, J. J.; Warias, R.; Lotter, C.; Mauritz, L.; Fuchs, P. J. W.; Ohla, S.; Zeitler, K.; Belder, D. Lab Chip 2016, 17, 76.

[152] Xia, H. L.; Hua, X.; Long, Y. T. Acta Chim. Sinica 2019, 77, 1164 (in Chinese). (夏海伦, 华金金, 龙亿涛, 化学学报, 2019, 77, 1164.)

[153] Jensen, K. F. AIChE J. 2017, 63, 858.

[154] Zhao, S. F.; Han, F.; Li, J. H.; Meng, X. Y.; Huang, W. P.; Cao, D. X.; Zhang, G. P.; Sun, R.; Wong, C. P. Small 2018, 14, 1800047.

[155] Pan, J.; Quan, L. N.; Zhao, Y. B.; Peng, W.; Murali, B.; Sarmah, S. P.; Yuan, M.; Sinatra, L.; Alyami, N. M.; Liu, J. K.; Yassitepe, E.; Yang, Z. Y.; Voznyy, O.; Comin, R.; Hedhili, M. N.; Mohammed, O. F.; Lu, Z. H.; Kim, D. H.; Sargent, E. H.; Bakr, O. M. Adv. Mater. 2016, 28, 8718 .

[156] Shen, Y.; Weeranoppanant, N.; Xie, L. S.; Chen, Y.; Lusardi, M. R.; Imbrogno, J.; Bawendi, M. G.; Jensen, K. F. Nanoscale 2017, 9 , 7703.

[157] Swain, B.; Hong, M. H.; Kang, L.; Kim, B. S.; Kim, N.-H.; Lee, C. G. Chem. Eng. J. 2017, 308, 311.

[158] Liu, G. T.; Wang, K.; Lu, Y. C.; Luo, G. S. Chem. Eng. J. 2014, 258, 34.

[159] Nightingale, A. M.; Bannock, J. H.; Krishnadasan, S. H.; O'Mahony, F. T. F.; Haque, S. A.; Sloan, J.; Drury, C.; McIntyre, R.; deMello, J. C. J. Mater. Chem. A 2013, 1, 4067.

(Cheng, B.) 